\title{
How can 3D Game Engines Create Photo-Realistic Interactive Architectural Visualizations?
}

\author{
Joseph Ratcliffe, Alain Simons, Bournemouth University \\ Fern Barrow \\ UK, BH12 5BB, Bournemouth \\ United Kingdom \\ i7218183@bournemouth.ac.uk | asimons@bournemouth.ac.uk
}

\begin{abstract}
Architectural Visualizations are the evolution from a once used technique of Architectural Rendering. Through the proliferation of modern technology, the industry has progressed by using more contemporary applications to produce three dimensional (3D) renders for the output of images and videos. Using such applications allows for "photo-realistic" visuals that have an uncanny representation to a real-life environment, for clients to visualize proposed buildings, which can offer both interior and exterior environments. However, such applications lack a key component that could extend the platform that the visualization industry currently uses. Through recent technological developments pertaining to game engines, virtual game environments can render high fidelity visuals in real-time whilst providing interactive elements for deployment to various devices. This paper aims to create and implement an alternative method to the conventional three-dimensional pre-rendered visualizations, using a 3D game engine that can provide an interactive based solution, distributed to a computer device, for both the industry and the end user to experience.
\end{abstract}

Keywords: Photo-realistic, Visualization, Architectural, Interactive, Real Time, Game Engine.

\section{Introduction}

Over the past decade, the need for 3D computer generated graphics has become ubiquitous in a variety of entertainment and industrial industries. They provide a certain kind of visual communication that allows for users or clients to understand the ideas being conveyed to them. In the architectural industry, being able to communicate such ideas has previously been dominated with the use of two dimensional (2D) renders. Although the traditional techniques are still being used in the industry today, by using 3D representations of a proposed office space, flat, etc. allows for visualization companies to create highly detailed environments that can aid in the promotion of their client's business by enticing potential buyers of properties. [1] 
Predominantly, within the industry, 3D rendering software is used. Applications, such as Chaos Theory's "V-Ray", allow for creators to render visualizations as frames to produce a video representation instead of having to run the environment in real-time. A benefit of such methods allows for greater visuals as the scene can have additional lighting, higher density models and high quality textures. On the other hand, such methods can cause the issue of slow render times.

Another area that needs to be considered for the visualization is the product. Commonly a short film will be produced from the 3D renders. Providing the client with a visual representation of how their prospective building will look, which can be used for construction and advertising purposes. Although this is useful to the client, they aren't interacting with the visualization. Having such interaction would allow for the client to be more engaged with the product and get a sense of how the user would act as if they were in the real-life scenario.

To achieve additional interaction an architectural visualization company could adopt the use of a 3D video game engine. Game engines are like that of a $3 \mathrm{D}$ rendering application however, the environments that are produced all run in real time and allow the user to walk through the environment as if they were there. Although there are slight visual discrepancies between the two, the benefit of a video game engine may outweigh the benefits of the $3 \mathrm{D}$ rendering application.

\section{Previous Research}

Architectural Visualizations are derived from a 2D technique called Architectural Rendering. However, as technology has progressed consumers / customers are wanting more interaction with architectural environments. Rosmani et al (2014) [2] addresses this view by proposing that many architectural visualization developers want to give viewers a more realistic environment based on $2 \mathrm{D}$ photos by creating a threedimensional adaptation.

Conventionally, within industry, computer-aided design (CAD) software is used. Per Szczepaniak (2016) [3], CAD software has become a required tool for companies that build complex machines and systems. An application such as Autodesk's "AutoCAD" functions as one of those tools. A report by "Research and Markets" (2016) [4] has forecasted that architectural rendering software such as Vray, Lumion and KeyShot as a market sector should grow by $29.37 \%$ during the period $2016-2020$, illustrating that there shows little sign of moving away from the traditional techniques.

As mentioned previously, within both rendered and real time solutions of a visualization or environment, lighting is an influential area in creating a realistic expectation of an environment. In a book by Mark Karlen et al (2012) [5], they discuss various elements on the basics of lighting design. Many of the principles that are mentioned within this source suggest that effective lighting should achieve "desired moods", "provide the proper amount of light" and "produce good colour". Many of 
these points directly coincide with how a visualization artist carries out their lighting systems within rendering engines and real time game engines.

Commonly architectural visualizations portray exterior designs or interior designs in 3D. In regards to interior design the use of colour can illustrate a certain life-style. Guangming Chen et al (2016) [6] relays this point by suggesting a colour scheme can "convey particular design philosophy and design a specific household style". This can be influenced by the colour of a room and furniture. For many visualization designers, they will choose a colour palette for the entirety of the project. For a modernistic approach, they may go for a "cool" colour palette or to have a classic effect go with a "warm" colour palette.

In industry, the previous conventional method of producing textures was through the means of artistic software packages such as Adobe Photoshop, within many institutions and disciplines, texturing has been produced using such digital software packages as Carol Faber (2007) [7] discusses in her paper. However, as technology has progressed, the process of texturing has also advanced. As the industry, has moved away from the previous conventional methods, a new texturing technique called "Procedural Texturing" has been introduced. This still incorporates the use of artistic software packages. Fundamentally, the procedural technique involves "code segments or algorithm that specify some characteristic of a computer-generated model or effect" David Ebert et al (2003). [8] This allows for artists to create textures within an efficient time frame.

Coinciding with the development of procedural texturing, a new method of material creation has been implemented called Physical Based Rendering (PBR). In a guide by Wes McDermott (2014) [9] expresses that "Light-Rays" are important to understand due to the way in which the light reacts with the surface of an object, that surface is governed by how the texture and material is made. In Volume 2 of the PBR guide by McDermott (2015) [10] PBR "is a method of shading and rendering that provides a more accurate representation of how light interacts with surfaces."

Currently game engines are being used to create a variety of solutions for different industries. As they contain powerful feature sets they become increasingly useful in creating highly detailed environments. Neal Bürger [11] considered the use of Unreal 3.5 and how it can be used to create a real time architectural visualization. In his paper, he summaries that the engine could output a solution that was "positively received".

A recent development in game engine technology has even provided the film industry with the means of creating high quality rendered characters and scenes with the use of real time game engines. The first company to use a game engine for this specific purpose was Industrial Light \& Magic (ILM) in the recent film "Star Wars: Rogue One". This information was gathered from a keynote by Tim Sweeney, CEO of Epic Games during GDC [12] about how some scenes within the film involving droid "K-2SO" were shot and rendered in real time within Unreal 4. Illustrating that the film industry has been looking to new methods to produce photo-realistic content without 
the use of the traditional rendering engines, companies such as Disney's Pixar have also been collaborating with Epic Games on the use of real-time game engines.

\section{Our Research}

Based upon research discovered within the literature review, refinement of software tools to implement a solution was determined. Three areas require bespoke software to create an implementation that relates to a traditional based visualization. These areas are: Modelling, Texturing and Game Engine. A modern approach to the production pipeline would be preferred as it's more efficient whilst still attaining detail. Each piece of software chosen is used within both video game and visualization industries as there is some overlap, however, the pipeline used will be more akin to the video game industry. Various elements have been influenced directly by research into the production process to architectural visualizations within game engines. Many of the theories covered within the literature review have been considered during the implementation. Colour theory, lighting theory are a selection of theories that have been adapted to create an architectural visualization fitting a specific life-style theme.

Interactive elements for the research have been produced using a node based scripting system within Unreal 4 called "Blueprints" that is a visual representation of code using $\mathrm{C}++$. These blueprints have been applied to door and lighting assets. Each asset contains their own bespoke blueprint scripts as they both function differently. Specifically, the scripting method that Epic Games has produced for their engine, is different from a competitive engine such as Unity, which offers a C\# programming language method.

The physical based rendering method of texture creation has influenced the production of in game materials, providing realistic properties comparative to an object within the real world.

Within packaged development builds, the command console can be accessed to provide data within the same testing environment that the test users receive. As frames per second (FPS) is an appropriate barometer of whether visualization is optimized to run on the test system hardware, "Stat FPS" can be used to display the FPS during run time. On the test system, the visualization produced a substantial 60 FPS, which provided a smooth experience for the user. 


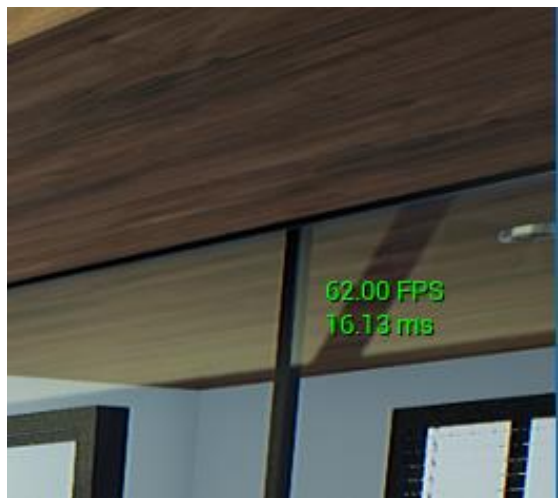

Fig 2. Frames Per Second data, during real time testing.

To ensure animations would initiate as intended, during simulation of the visualization, within the actor blueprint an event graph displays information to illustrate if the blueprint is entering a node correctly.

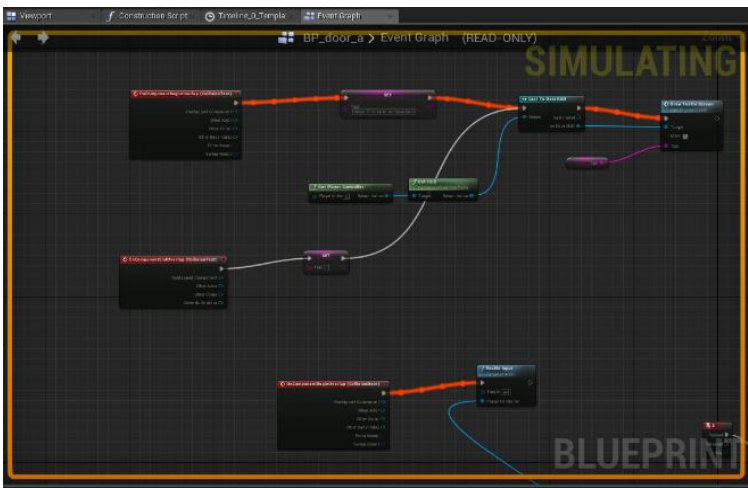

Fig 3. Door actor blueprint, event graph

In addition to checking correct animation, revealing the collision boxes in development builds, confirmed that collision triggers were placed in correct areas to the player.

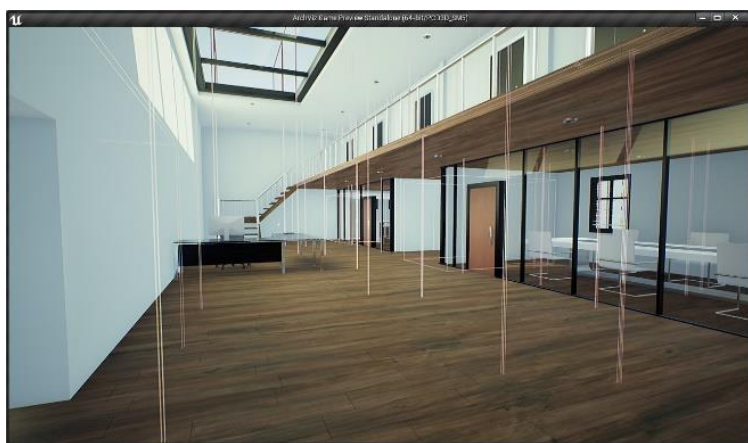

Fig 4. Rendered collision boxes, testing animation triggers 
As correct lightmap resolution is critical to solving shadow irregularities within the visualization, during construction, the "Light Map Density" optimization view provided information as to whether an asset contained the "optimal" lightmap resolution.

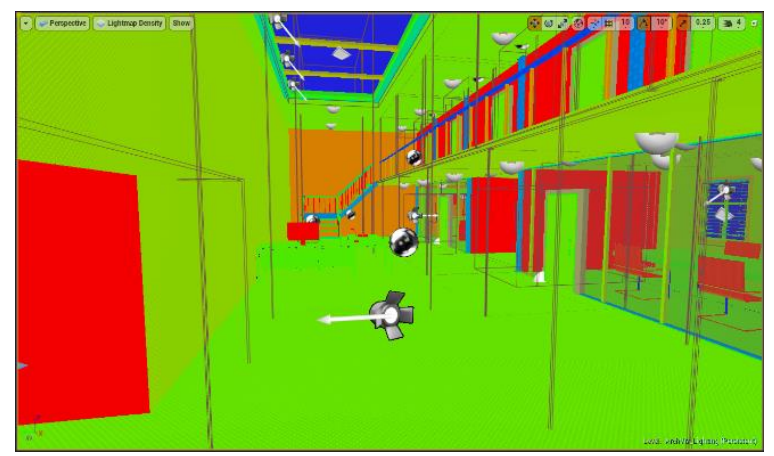

Fig 5. Lightmap Density view mode

Blue coloured meshes allude to the asset perhaps needing a higher resolution, green is the ideal resolution and red is over compensating, however a balance is required as higher resolution lightmaps are more expensive in real-time. Most smaller modular sections have an exaggerated lightmap to provide sufficient shadows.

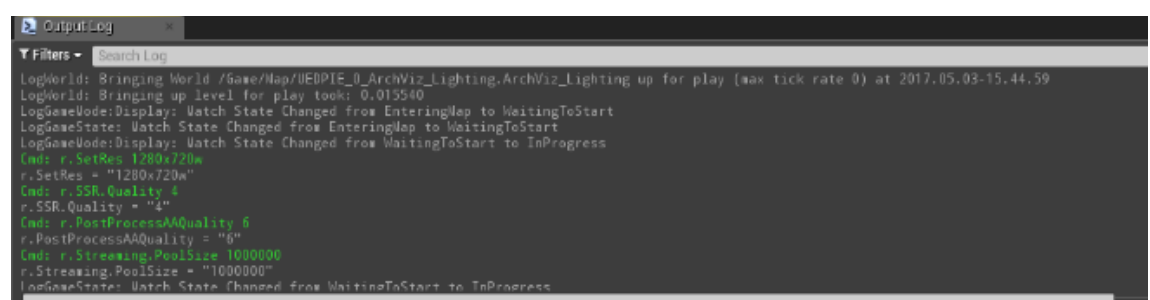

Fig 6. Real-time output log of executed console commands

The last method to check whether "under the hood" commands execute, an output log window provides data on the specified commands, amongst other pieces of data. This window can only be accessed within the Unreal editor.

Optimization of the solution has been extensive for the solution to work on multiple computer systems whilst still attempting to maintain standards of an industry standard visualization. Most real-time architectural visualizations require computational power that most users won't have access to, this is due to lighting calculations, scene loading and other elements all having to run in real-time when using a game engine.

However, due to the visualization running natively on a computer system rather than as a web based application, a web browser can't load detailed scenes in an efficient time compared to the real-time executable application. As Liu Xiaojun et al (2016) [13] imply within their paper, Web3D solutions can't render complex interactive scenes. 


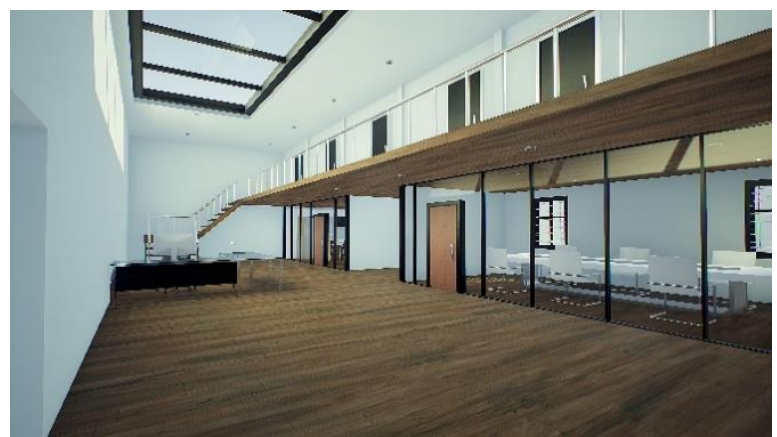

Fig 1. A view on the presented test visualization

Results have been gathered by providing an executable Unreal 4 file and a video of a traditional rendered visualization which has been produced within KeyShot. These files were distributed through a Google Drive link and face-to-face. The latter testing method was conducted within a business complex on a laptop with a 970M Nvidia Graphics Card.

\section{Results}

To answer the question of "How can 3D Game Engines create Photo-Realistic Interactive Architectural Visualizations?", data pertaining to both technical and emotional standpoints are needed. Due to the primary target audience gathered are adults that have had experience in purchasing home and/or commercial real estate, the survey questions needed to be delivered in an understandable manner as the audience may not have the required expertise to understand how the visualization works.

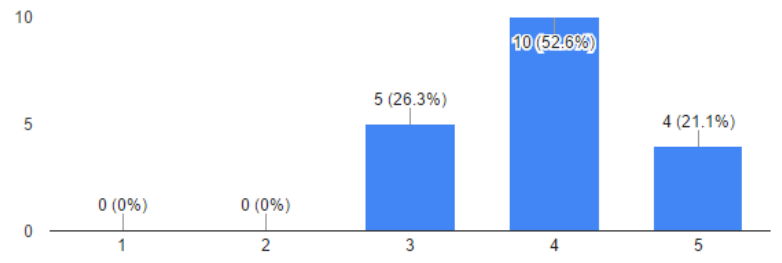

Fig 7. Do you think the Real-time visualization's visual quality compare to that of the rendered visualization?

Results suggested that users felt that the quality was close to comparing to that of the rendered version, most responses of the survey suggested there were more objects within the rendered version which gave greater visual quality. However, many users preferred the sense of scale that can be conveyed from an interactive version as all elements are built to a 1:1 scale ratio to mimic a real-life viewing. 


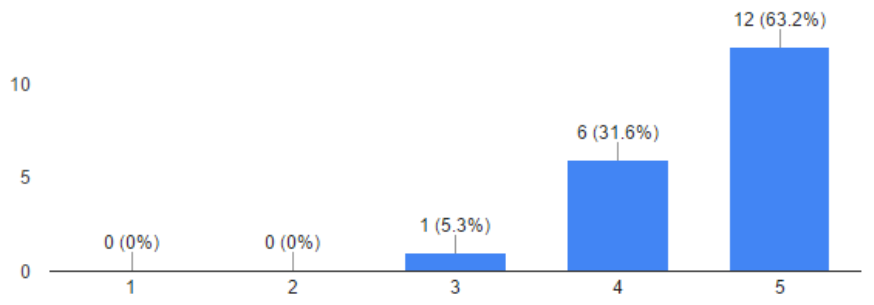

Fig 8. Does the additional interaction (e.g. Open doors, walk through) benefit the visualization?

$63.2 \%$ of users agree on interactive elements provide a benefit within the interactive solution, however some users were having issues in the control of their actions as elements such as doors could only be opened when close to the door and found that some of the collisions within the solution were frustrating.

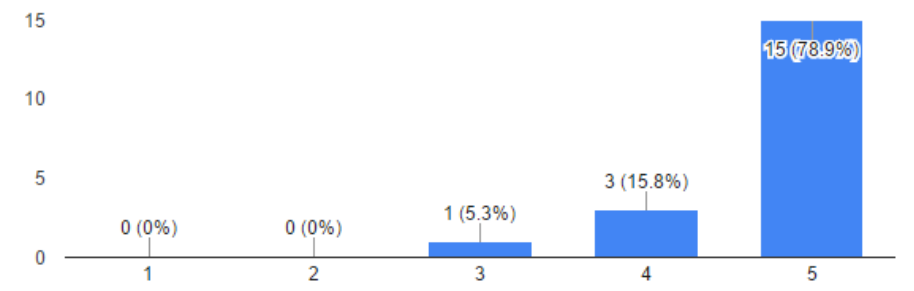

Fig 9. Did you prefer the video presentation over the interactive walk through?

Although with these slight frustrations, interaction still provides a benefit. Almost $80 \%$ of users preferred the interactive walkthrough greatly over the video walkthrough. A frequent discussion as to why users preferred this method of delivery was largely down to being able to view everything that they wanted. Whereas the video version can only show specific portions of the visualization. Understanding of scale, floor layout among other aspects influenced their decision.

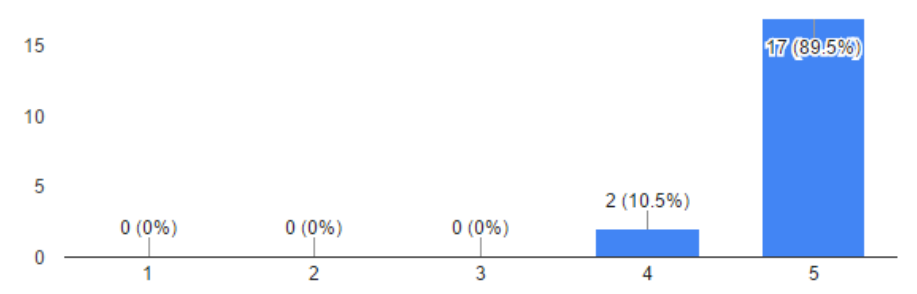

Fig 10. Do you think interactive walk through's, have a place in the future?

An overwhelming number of users sided with interactive visualizations having a place within the visualization industry. Although suggestions of a better methods with other technologies such as Virtual Reality and perhaps utilising mobile technologies further were given. 
On the contrary to information given from "Research and Markets" the industry suggesting that rendering software sales will rise till 2020, real-time game engine software such as Unreal 4, can provide other types of experiences that may well eclipse the use of rendering engines in the future.

\section{Conclusion}

A real-time game engine solution isn't distant to the rendered version in terms of visual quality, although one may conclude due to the slight limitation in achieving an exact comparison to the rendered version, would hinder the audience. Results illustrated by the outcome of both the third and fourth question asked, the audience of such realtime visualizations are interested with an interactive over the traditional solution.

For certain elements to be interactive, a sacrifice should be made in lighting quality by using moveable or stationary lights as they can be activated in runtime. However, as evident from results, the interactivity doesn't sacrifice from visual quality to a large extent.

Although, there are improvements to be made in the delivery of interactive based solutions with the inclusion of other technologies such as Virtual Reality, Augmented Reality and Mobile technologies.

\section{References}

1. Village Features 3D / Hale, J., 2015. What is Architectural Visualization?[online]. Bluffton, South Carolina. Available from: https://www.villagefeatures3d.com/blog/what-is-architectural-visualization [Accessed 10 March 2017].

2. Rosmani, AFR., Mazlan, UHM., Ahmad, SZA. and Apendi, AAMKA., 2014. Developing an Architectural Visualization using 3D for Photo Tourism [online]. IEEE 2014 International Conference on Computer, Communication, and Control Technology, Langkawi, Kedah, Malaysia 2-4 September 2014. Arau: Universiti Teknologi MARA. Available from: http://ieeexplore.ieee.org/stamp/stamp.jsp?arnumber $=6914220$ [Accessed 7th November 2016].

3. Szczepaniak, PS., 2014. From 3D CAD to 3D Rendering. Machine Design, 86 (8), $54-57$.

4. Research and Markets, RAM., 2016. Visualization and 3D Rendering Software Market 2016-2020 - High-End Video Games, Architectural and Product Visualization, Training Simulation, Marketing and Advertisement - Research and
Markets
[online].
Dublin.
Available
from:

http://www.businesswire.com/news/home/20160331005465/en// [Accessed 7 November 2016]. 
5. Karlen, M., Benya, J., 2017. Lighting Design Basics. $3^{\text {rd }}$ Edition. Wiley.

6. Chen, G., Li, G., Nie, Y., Xian, C., Mao, A., 2016. Stylistic indoor colour design via Bayesian network. Computers \& Graphics [online], 60, 34-45.

7. Faber, CH., 2007. Traditional Material Use in A Digital World: A Creative Design Methodology. International Association of Societies of Design Research [online].

8. Ebert, DS., 2003. Texturing \& Modeling: A Procedural Approach. $3^{\text {rd }}$ Edition. Morgan Kaufmann.

9. McDermott, WM., 2014. The Comprehensive PBR Guide by Allegorithmic - vol 1 [PDF]. Allegorithmic.

10. McDermott, WM., 2015. The Comprehensive PBR Guide by Allegorithmic - vol 2 [PDF]. Allegorithmic.

11. Bürger,NB.,2010.Realtime Interactive Architectural Visualization using Unreal Engine 3.5. 2017[online].

12. Epic Games, EG., 2017. Quality and Innovation Take Center Stage During 'State of Unreal' at GDC 2017[online]. Cary, North Carolina. Available from: https://www.unrealengine.com/blog/quality-and-innovation-take-center-stageduring-state-of-unreal-at-gdc-2017 [Accessed 10 March 2017].

13. Liu, X., Xie, N., Tang, K., Jia, J., 2016. Lightweighting for Web3D visualization of large-scale BIM scenes in real-time. Graphical Models, 88, 40-56. 